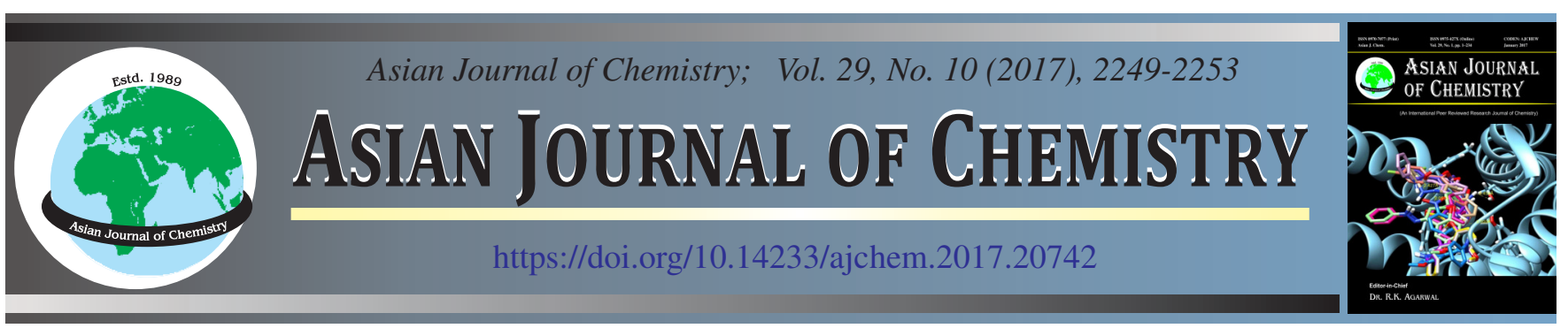

\title{
Synthesis and in vitro Study of Novel Thiophenyl Benzoimidazolyl Benzamides, Acrylamides and Propionamides as Antimicrobial Agents
}

\author{
M. Prasada Reddy, Ravi Chandra Babu*, M. Sudhalakshmi and A. Siva Rami Reddy
}

Department of Chemistry, Institute of Science, GITAM University, Visakhapatnam-530 045, India

*Corresponding author: E-mail: prasadreddy.m83@gmail.com

Keywords: Solid state reaction, Thiophenyl acids, Thiophenyl benzimidazoles, Antibacterial activity, Antifungal activity.

\section{INTRODUCTION}

A large number of heterocyclic compounds play a vital role as drugs and pharmaceutical agents. The benzoimidazole moiety is a core structure in various synthetic pharmaceuticals displaying a broad spectrum of biological activity including antiulcer, antitumor, antiviral and antibacterial effects [1-7]. Substituted 2-amino-ethylthiophenes also constitute an important class of heterocyclic compounds possessing diverse biological activities [8-11]. In addition to being bioactive substances, these compounds find wide applications in synthesis. Due to the continuing increase of antibacterial resistance and the lack of novel antibiotics in the past several decades, it is of great interest to discover novel antibiotics that could combat the antibacterial resistance. This has given an impetus for the synthesis of new ethylthiophenyl benzoimidazolyl benzamides, acrylamides and propionamides and evaluation of their biological significance and toxicity.

Thus, the development of practical and convenient method for the construction of nitrogen containing heterocycles is an important goal. In continuation of our work on substituted 2-amino-ethylthiophenes, the present investigation reports the synthesis and antimicrobial activity of ethylthiophenyl benzoimidazolyl benzamides, acrylamides and propionamides.

\section{EXPERIMENTAL}

All the reagents and solvents were used as purchased without further purification melting points were determined in open capillary on a cintex melting point apparatus and are uncorrected. Purity of the compounds was checked by TLC. TLC analyses performed on precoated silicagel (E. Merck kieselgel $60 \mathrm{~F}_{254}$ ) plates and visualization was done by exposing to iodine vapour. IR (KBr) spectra were recorded on a PerkinElmer 282 instrument; ${ }^{1} \mathrm{H}$ NMR spectra on a Varian EM-390 spectrometer using TMS as internal reference; and mass spectra on a Varian MAT CH-7 instrument at $70 \mathrm{eV}$. C, $\mathrm{H}$ and $\mathrm{N}$ analyses were carried out on Carlo Erba 106 and Perkin-Elmer analysers.

2-[(4-Benzoyl-5-ethylthiophen-2-yl)carbamoyl]benzoic acid (2): Substituted ethyl-2-aminothiophene (1) (0.01 mol) and phthalic anhydride $(0.01 \mathrm{~mol})$ were mixed well in mortar and pestle. The intimate mixture was ground occasionally for $1 \mathrm{~h}$ at room temperature. The reaction-mixture (monitored with TLC) was kept at ambient temperature for another $1 \mathrm{~h}$ for completion of the reaction. The resulting solid was extracted with aqueous sodium bicarbonate solution and filtered. The clear filtrate on acidification gave the solid, which was recrystallized from ethyl alcohol to get white crystals.

2-[(4-Benzoyl-5-ethylthiophen-2-yl)carbamoyl]-acrylic acid (4) and propionic acid (6) were prepared similarly following the same reaction conditions as described for compound 2.

2-(1H-Benzo[d]imidazol-2-yl)- $N$-(4-benzolyl-5-ethylthiophene-2-yl)-benzamide (3a-c): 2-[(4-Benzoyl-5-ethylthiophen-2-yl)carbamoyl]benzoic acid (2) (0.01 mol) and $o$-phenylenediamine $(0.01 \mathrm{~mol})$ were taken in pyridine $(30$ $\mathrm{mL}$ ) and the contents were refluxed for $5 \mathrm{~h}$. The contents after cooling were poured into crushed ice, separated solid was 
filtered, washed with water. Recrystallization was done with ethyl acetate and benzene.

3-(1H-benzo[ $d$ ]imidazol-2-yl)- $N$-(5-methyl-3-isoxazolyl)acrylamides (5a-c) and propionamides (7a-c) were prepared similarly following the same reaction conditions as described for compounds 3a-c.

\section{Physical and spectral data}

2-[(4-Benzoyl-5-ethylthiophen-2-yl)carbamoyl]benzoic acid (2): Yield: $90 \%$; m.p.: $165-167^{\circ} \mathrm{C}$; IR $\left(\mathrm{KBr}, \mathrm{v}_{\max }, \mathrm{cm}^{-1}\right)$ : 3024 (Ar-H), 2962 (C-H, CH$), 1740$ (C=O), 685 (C-S), 1123 (C-N), $3250(\mathrm{O}-\mathrm{H}), 1642(\mathrm{C}=\mathrm{C})$; ${ }^{1} \mathrm{H}$ NMR (300 MHz, DMSO): $\delta 1.25(\mathrm{t}, 3 \mathrm{H}), 2.87(\mathrm{q}, 2 \mathrm{H}), 6.07(\mathrm{~s}, 1 \mathrm{H}), 7.33(\mathrm{~d}, 1 \mathrm{H}), 7.34(\mathrm{~d}$, $1 \mathrm{H}), 7.48(\mathrm{t}, 1 \mathrm{H}), 7.49(\mathrm{t}, 1 \mathrm{H}), 7.55(\mathrm{t}, 1 \mathrm{H}), 7.89(\mathrm{~d}, 1 \mathrm{H}), 7.95$ $(\mathrm{t}, 1 \mathrm{H}), 7.99(\mathrm{t}, 1 \mathrm{H}), 8.45(\mathrm{~d}, 1 \mathrm{H}), 9.23(\mathrm{~s}, 1 \mathrm{H}), 11.2(\mathrm{~s}, 1 \mathrm{H})$; MS $m / z 379.43\left(\mathrm{M}^{+}\right)$; Elemental analysis (\%) calculated for $\mathrm{C}_{21} \mathrm{H}_{17} \mathrm{NO}_{4} \mathrm{~S}$ : C-66.47, H-4.52, N-3.69, O-16.87, S-8.45. Found C-65.9, H-4.23, N-3.55, O-16.24, S-8.12.

2-(1H-Benzo[d]imidazol-2-yl)- $N$-(4-benzoyl-5-ethylthiophen-2-yl)benzamide (3a): Yield: 87 \%; m.p.: 245-247 ${ }^{\circ} \mathrm{C}$; IR (KBr, $\left.v_{\max }, \mathrm{cm}^{-1}\right): 3029(\mathrm{Ar}-\mathrm{H}), 2965\left(\mathrm{C}-\mathrm{H}, \mathrm{CH}_{2}\right), 1760$ $(\mathrm{C}=\mathrm{O}), 689(\mathrm{C}-\mathrm{S}), 1123(\mathrm{C}-\mathrm{N}), 1648(\mathrm{C}=\mathrm{C}) ;{ }^{1} \mathrm{H}$ NMR $(300$ MHz, DMSO): $\delta 1.25$ (t, 3H), 2.87 (q, 2H), 5.0 (s, 1H), 6.16 $(\mathrm{s}, 1 \mathrm{H}), 7.23(\mathrm{t}, 1 \mathrm{H}), 7.23(\mathrm{t}, 1 \mathrm{H}), 7.34(\mathrm{~d}, 1 \mathrm{H}), 7.35(\mathrm{~d}, 1 \mathrm{H})$, $7.48(\mathrm{t}, 1 \mathrm{H}), 7.45(\mathrm{t}, 1 \mathrm{H}), 7.45(\mathrm{t}, 1 \mathrm{H}), 7.57(\mathrm{t}, 1 \mathrm{H}), 7.57(\mathrm{~d}$, $1 \mathrm{H}), 7.59(\mathrm{~d}, 1 \mathrm{H}), 7.76(\mathrm{t}, 1 \mathrm{H}), 7.97(\mathrm{~d}, 1 \mathrm{H}), 8.09(\mathrm{~d}, 1 \mathrm{H})$, 9.15 (s, 1H); MS m/z $451.54\left(\mathrm{M}^{+}\right)$; Elemental analysis (\%) calculated for $\mathrm{C}_{27} \mathrm{H}_{21} \mathrm{~N}_{3} \mathrm{O}_{2} \mathrm{~S}: \mathrm{C}, 71.82 ; \mathrm{H}, 4.69 ; \mathrm{N}, 9.31 ; \mathrm{O}$, 7.09; S, 7.10. Found C-70.56, H-4.52, N-8.25, O-6.85, S-6.98.

$\mathrm{N}$-[5-Ethyl-4-(phenylcarbonothioyl)thiophen-2-yl]-2(4-nitro-1H-benzo[d]imidazol-2-yl)benzamide (3b): Yield: $78 \%$; m.p.: 220-222 ${ }^{\circ} \mathrm{C}$; IR (KBr, $\left.v_{\max }, \mathrm{cm}^{-1}\right)$ : 3035 (Ar-H), 2958 (C-H, $\left.\mathrm{CH}_{2}\right), 1750$ (C=O), 683 (C-S), 1135 (C-N), 1658 $(\mathrm{C}=\mathrm{C}), 1524(\mathrm{~N}-\mathrm{O}) ;{ }^{1} \mathrm{H}$ NMR $(300 \mathrm{MHz}, \mathrm{DMSO}): \delta 1.26(\mathrm{t}$, $3 \mathrm{H}), 2.89$ (q, 2H), $5.25(\mathrm{~s}, 1 \mathrm{H}), 6.26(\mathrm{~s}, 1 \mathrm{H}), 7.49(\mathrm{t}, 1 \mathrm{H}), 7.47$ $(\mathrm{d}, 1 \mathrm{H}), 7.38(\mathrm{~d}, 1 \mathrm{H}), 7.37(\mathrm{~d}, 1 \mathrm{H}), 7.41(\mathrm{t}, 1 \mathrm{H}), 7.48(\mathrm{t}, 1 \mathrm{H})$, $7.49(\mathrm{t}, 1 \mathrm{H}), 7.57(\mathrm{t}, 1 \mathrm{H}), 7.96(\mathrm{~d}, 1 \mathrm{H}), 7.77(\mathrm{t}, 1 \mathrm{H}), 7.93(\mathrm{~d}$, $1 \mathrm{H}), 8.19(\mathrm{~d}, 1 \mathrm{H}), 9.85(\mathrm{~s}, 1 \mathrm{H})$; MS m/z $512.60\left(\mathrm{M}^{+}\right)$; Elemental analysis (\%) calculated for $\mathrm{C}_{27} \mathrm{H}_{20} \mathrm{~N}_{4} \mathrm{O}_{3} \mathrm{~S}_{2}$ : C-63.26, H-3.93, N-10.93, O-9.36, S-12.51. Found C-62.52, H-3.82, N-9.98, O-9.08, S-11.95.

$\mathrm{N}$-(4-Benzoyl-5-ethylthiophen-2-yl)-2-(5,6-dichloro$\mathbf{1 H}$-benzo[d]imidazol-2-yl)benzamide (3c): Yield: $82 \%$; m.p.: 238-240 ${ }^{\circ} \mathrm{C}$; IR (KBr, $\left.v_{\max }, \mathrm{cm}^{-1}\right): 3025$ (Ar-H), 2968 $\left(\mathrm{C}-\mathrm{H}, \mathrm{CH}_{2}\right), 1740(\mathrm{C}=\mathrm{O}), 689(\mathrm{C}-\mathrm{S}), 1185(\mathrm{C}-\mathrm{N}), 1668(\mathrm{C}=\mathrm{C})$, 792 (C-Cl); ${ }^{1} \mathrm{H}$ NMR (300 MHz, DMSO): $\delta 1.28$ (t, 3H), 2.79 $(\mathrm{q}, 2 \mathrm{H}), 5.35(\mathrm{~s}, 1 \mathrm{H}), 6.36(\mathrm{~s}, 1 \mathrm{H}), 7.47(\mathrm{~d}, 1 \mathrm{H}), 7.38(\mathrm{~d}, 1 \mathrm{H})$, $7.37(\mathrm{~d}, 1 \mathrm{H}), 7.41(\mathrm{t}, 1 \mathrm{H}), 7.49(\mathrm{t}, 1 \mathrm{H}), 7.57(\mathrm{t}, 1 \mathrm{H}), 7.96(\mathrm{~d}$, $1 \mathrm{H}), 7.77(\mathrm{t}, 1 \mathrm{H}), 7.93(\mathrm{~d}, 1 \mathrm{H}), 8.45(\mathrm{~s}, 1 \mathrm{H}), 8.54(\mathrm{~s}, 1 \mathrm{H}), 9.85$ $(\mathrm{s}, 1 \mathrm{H}) ; \mathrm{MS} \mathrm{m} / 2520.43\left(\mathrm{M}^{+}\right)$; Elemental analysis (\%) calculated for $\mathrm{C}_{27} \mathrm{H}_{19} \mathrm{~N}_{3} \mathrm{O}_{2} \mathrm{SCl}_{2}$ : C-62.31, H-3.68, Cl-13.62, N-8.07, O6.15, S-6.16. Found C-62.02, H-3.52, N-7.95, O-5.98, S-5.95, Cl-13.02.

4-[(4-Benzoyl-5-ethylthiophen-2-yl]amino)-4-oxobut2-enoic acid (4): Yield: $88 \%$; m.p.: $155-157{ }^{\circ} \mathrm{C}$; IR (KBr, $\left.v_{\max }, \mathrm{cm}^{-1}\right): 3032(\mathrm{Ar}-\mathrm{H}), 2978\left(\mathrm{C}-\mathrm{H}, \mathrm{CH}_{2}\right), 1730(\mathrm{C}=\mathrm{O}), 679$ (C-S), $1165(\mathrm{C}-\mathrm{N}), 1658(\mathrm{C}=\mathrm{C}), 3325(\mathrm{~N}-\mathrm{H}), 3200(\mathrm{O}-\mathrm{H}) ;{ }^{1} \mathrm{H}$ NMR (300 MHz, DMSO): $\delta 1.23(\mathrm{t}, 3 \mathrm{H}), 2.67$ (q, 2H), 6.22 $(\mathrm{s}, 1 \mathrm{H}), 6.51(\mathrm{~d}, 1 \mathrm{H}), 6.82(\mathrm{~d}, 1 \mathrm{H}), 7.69(\mathrm{t}, 1 \mathrm{H}), 7.68(\mathrm{t}, 1 \mathrm{H})$, $7.75(\mathrm{t}, 1 \mathrm{H}), 7.95(\mathrm{~d}, 1 \mathrm{H}), 7.99(\mathrm{~d}, 1 \mathrm{H}), 9.20(\mathrm{~s}, 1 \mathrm{H}), 11.0(\mathrm{~s}$, $1 \mathrm{H})$; MS m/z $329.37\left(\mathrm{M}^{+}\right)$; Elemental analysis (\%) calculated for $\mathrm{C}_{17} \mathrm{H}_{15} \mathrm{NO}_{4} \mathrm{~S}$ : C-61.99, H-4.59, N-4.25, O-19.43, S-9.74. Found C-61.02, H-4.02, N-4.03, O-18.56, S-9.02.

3-(1H-Benzo[ $d]$ imidazol-2-yl)- $N$-(4-benzoyl-5-ethylthiophen-2-yl)acrylamide (5a): Yield: $79 \%$; m.p.: 240-242 ${ }^{\circ} \mathrm{C}$; IR $\left(\mathrm{KBr}, v_{\max }, \mathrm{cm}^{-1}\right)$ : $3063(\mathrm{Ar}-\mathrm{H}), 2968\left(\mathrm{C}-\mathrm{H}, \mathrm{CH}_{2}\right), 1745$ $(\mathrm{C}=\mathrm{O}), 689(\mathrm{C}-\mathrm{S}), 1175(\mathrm{C}-\mathrm{N}), 1678(\mathrm{C}=\mathrm{C}), 3330(\mathrm{~N}-\mathrm{H}) ;{ }^{1} \mathrm{H}$ NMR (300 MHz, DMSO): $\delta 1.25(\mathrm{t}, 3 \mathrm{H}), 2.87(\mathrm{q}, 2 \mathrm{H}), 5.0(\mathrm{~s}$, $1 \mathrm{H}), 6.22(\mathrm{~s}, 1 \mathrm{H}), 6.51(\mathrm{~d}, 1 \mathrm{H}), 6.92(\mathrm{~d}, 1 \mathrm{H}), 7.32(\mathrm{t}, 1 \mathrm{H}), 7.32$ $(\mathrm{t}, 1 \mathrm{H}), 7.50(\mathrm{~d}, 1 \mathrm{H}), 7.53(\mathrm{~s}, 1 \mathrm{H}), 7.69(\mathrm{t}, 1 \mathrm{H}), 7.68(\mathrm{t}, 1 \mathrm{H})$, $7.75(\mathrm{t}, 1 \mathrm{H}), 7.95$ (d, 1H), $7.99(\mathrm{~d}, 1 \mathrm{H}), 9.20(\mathrm{~s}, 1 \mathrm{H}) ; \mathrm{MS} \mathrm{m} / z$ $401.48\left(\mathrm{M}^{+}\right)$; Elemental analysis $(\%)$ calculated for $\mathrm{C}_{23} \mathrm{H}_{19} \mathrm{~N}_{3} \mathrm{O}_{2} \mathrm{~S}$ : C-68.81, H-4.77, N-10.47, O-7.97, S-7.99. Found C-68.12, H-4.12, N-9.98, O-7.02, S-7.12.

$\mathrm{N}$-(4-Benzoyl-5-ethylthiophen-2-yl)-3-(4-nitro-1Hbenzo $[\boldsymbol{d}]$ imidazol-2-yl)acrylamide (5b): Yield: $82 \%$; m.p.: 205-207 ${ }^{\circ} \mathrm{C}$; IR (KBr, $\left.v_{\max }, \mathrm{cm}^{-1}\right): 3053(\mathrm{Ar}-\mathrm{H}), 2958(\mathrm{C}-\mathrm{H}$, $\left.\mathrm{CH}_{2}\right), 1745(\mathrm{C}=\mathrm{O}), 689(\mathrm{C}-\mathrm{S}), 1165(\mathrm{C}-\mathrm{N}), 1688(\mathrm{C}=\mathrm{C}), 3320$ $(\mathrm{N}-\mathrm{H}), 1525(\mathrm{~N}-\mathrm{O})$; 1H NMR (300 MHz, DMSO): $\delta 1.21(\mathrm{t}$, $3 \mathrm{H}), 2.67$ (q, 2H), $5.0(\mathrm{~s}, 1 \mathrm{H}), 6.22(\mathrm{~s}, 1 \mathrm{H}), 6.51(\mathrm{~d}, 1 \mathrm{H}), 7.12$ $(\mathrm{d}, 1 \mathrm{H}), 7.56(\mathrm{t}, 1 \mathrm{H}), 7.53(\mathrm{~s}, 1 \mathrm{H}), 7.69(\mathrm{t}, 1 \mathrm{H}), 7.68(\mathrm{t}, 1 \mathrm{H})$, $7.75(\mathrm{t}, 1 \mathrm{H}), 7.95(\mathrm{~d}, 1 \mathrm{H}), 7.99(\mathrm{~d}, 1 \mathrm{H}), 7.99(\mathrm{~d}, 1 \mathrm{H}), 8.15$ (d, 1H), $9.20(\mathrm{~s}, 1 \mathrm{H})$; $\mathrm{MS} \mathrm{m} / z, 446.48\left(\mathrm{M}^{+}\right)$; Elemental analysis (\%) calculated for $\mathrm{C}_{23} \mathrm{H}_{18} \mathrm{~N}_{4} \mathrm{O}_{4} \mathrm{~S}$ : C-61.87, H-4.06, N-12.55, O-14.33, S-7.18. Found C-60.89, H-4.01, N-12.03, O-13.98, S-7.12.

$\mathrm{N}$-(4-Benzoyl-5-ethylthiophen-2-yl)-3-(5,6-dichloro$\mathbf{1 H}$-benzo $[\boldsymbol{d}]$ imidazol-2-yl)acrylamide $(\mathbf{5 c})$ : Yield: $78 \%$; m.p.: 218-220 ${ }^{\circ} \mathrm{C}$; IR (KBr, $\left.v_{\max }, \mathrm{cm}^{-1}\right)$ : 3048 (Ar-H), 2928 (C-H, $\left.\mathrm{CH}_{2}\right), 1735(\mathrm{C}=\mathrm{O}), 679(\mathrm{C}-\mathrm{S}), 1155(\mathrm{C}-\mathrm{N}), 1678(\mathrm{C}=\mathrm{C})$, $3310(\mathrm{~N}-\mathrm{H}), 685$ (C-Cl); ${ }^{1} \mathrm{H}$ NMR (300 MHz, DMSO): $\delta 1.18$ $(\mathrm{t}, 3 \mathrm{H}), 2.47(\mathrm{q}, 2 \mathrm{H}), 5.10(\mathrm{~s}, 1 \mathrm{H}), 6.32(\mathrm{~s}, 1 \mathrm{H}), 6.51(\mathrm{~d}, 1 \mathrm{H})$, $7.09(\mathrm{~d}, 1 \mathrm{H}), 7.66(\mathrm{t}, 1 \mathrm{H}), 7.55(\mathrm{~s}, 1 \mathrm{H}), 7.89(\mathrm{t}, 1 \mathrm{H}), 7.88(\mathrm{t}$, $1 \mathrm{H}), 7.99(\mathrm{~d}, 1 \mathrm{H}), 8.15(\mathrm{~d}, 1 \mathrm{H}), 8.35(\mathrm{~s}, 1 \mathrm{H}), 8.45(\mathrm{~s}, 1 \mathrm{H})$, $9.20(\mathrm{~s}, 1 \mathrm{H})$; MS m/z $470.37\left(\mathrm{M}^{+}\right)$; Elemental analysis (\%) calculated for $\mathrm{C}_{23} \mathrm{H}_{17} \mathrm{~N}_{3} \mathrm{O}_{2} \mathrm{SCl}_{2}$ : C-58.73, H-3.64, N-8.93, O6.80, S-6.82, Cl-15.07. Found C-57.93, H-3.26, N-8.03, O6.12, S-6.01, Cl-14.02.

4-[(4-Benzoyl-5-ethylthiophen-2-yl)amino]-4-oxobutanoic acid (6): Yield: $75 \%$; m.p.: 190-192 ${ }^{\circ} \mathrm{C}$; IR (KBr, $v_{\max }$, $\left.\mathrm{cm}^{-1}\right)$ : 3036 (Ar-H), $2968\left(\mathrm{C}-\mathrm{H}, \mathrm{CH}_{2}\right), 1720(\mathrm{C}=\mathrm{O}), 689(\mathrm{C}-$ S), $1175(\mathrm{C}-\mathrm{N}), 1698(\mathrm{C}=\mathrm{C}), 3335(\mathrm{~N}-\mathrm{H}), 3230(\mathrm{O}-\mathrm{H}) ;{ }^{1} \mathrm{H}$ NMR (300 MHz, DMSO): $\delta 1.29(\mathrm{t}, 3 \mathrm{H}), 2.44(\mathrm{t}, 2 \mathrm{H}), 2.68(\mathrm{t}$, 2H), $2.67(\mathrm{q}, 2 \mathrm{H}), 6.82(\mathrm{~d}, 1 \mathrm{H}), 7.69(\mathrm{t}, 1 \mathrm{H}), 7.68(\mathrm{t}, 1 \mathrm{H}), 7.75$ $(\mathrm{t}, 1 \mathrm{H}), 7.95(\mathrm{~d}, 1 \mathrm{H}), 7.99(\mathrm{~d}, 1 \mathrm{H}), 9.20(\mathrm{~s}, 1 \mathrm{H}), 11.9(\mathrm{~s}, 1 \mathrm{H})$; MS m/z $331.39\left(\mathrm{M}^{+}\right)$; Elemental analysis (\%) calculated for $\mathrm{C}_{17} \mathrm{H}_{17} \mathrm{NO}_{4} \mathrm{~S}$ : C-61.61, H-5.17, N-4.23, O-19.31, S-9.68. Found C-62.02, H-3.52, N-7.95, O-5.98, S-5.95.

3-(1H-Benzo[d]imidazol-2-yl)- $N$-(4-benzoyl-5-ethylthiophen-2-yl)propanamide (7a): Yield: $75 \%$; m.p.: 230$232{ }^{\circ} \mathrm{C}$; IR (KBr, $\left.v_{\max }, \mathrm{cm}^{-1}\right)$ : $3063(\mathrm{Ar}-\mathrm{H}), 2968\left(\mathrm{C}-\mathrm{H}, \mathrm{CH}_{2}\right)$, $1745(\mathrm{C}=\mathrm{O}), 689$ (C-S), $1175(\mathrm{C}-\mathrm{N}), 1678(\mathrm{C}=\mathrm{C}), 3330(\mathrm{~N}-$ $\mathrm{H}) ;{ }^{1} \mathrm{H}$ NMR (300 MHz, DMSO): $\delta 1.25$ (t, 3H), 2.87 (q, 2H), $2.98(\mathrm{t}, 2 \mathrm{H}), 2.89(\mathrm{t}, 2 \mathrm{H}), 5.0(\mathrm{~s}, 1 \mathrm{H}), 6.22(\mathrm{~s}, 1 \mathrm{H}), 7.32(\mathrm{t}$, $1 \mathrm{H}), 7.32(\mathrm{t}, 1 \mathrm{H}), 7.50(\mathrm{~d}, 1 \mathrm{H}), 7.53(\mathrm{~s}, 1 \mathrm{H}), 7.69(\mathrm{t}, 1 \mathrm{H}), 7.68$ (t, 1H), $7.75(\mathrm{t}, 1 \mathrm{H}), 7.95(\mathrm{~d}, 1 \mathrm{H}), 7.99(\mathrm{~d}, 1 \mathrm{H}), 9.20(\mathrm{~s}, 1 \mathrm{H})$; 
MS $m / z 403.50\left(\mathrm{M}^{+}\right)$; Elemental analysis (\%) calculated for $\mathrm{C}_{23} \mathrm{H}_{21} \mathrm{~N}_{3} \mathrm{O}_{2} \mathrm{~S}$ : C-68.46, H-5.25, N-10.41, O-7.93, S-7.95. Found C-68.12, H-4.12, N-9.98, O-7.02, S-7.12.

$\mathrm{N}$-(4-Benzoyl-5-ethylthiophen-2-yl)-3-(4-nitro-1Hbenzo[d] imidazol-2-yl)propanamide (7b): Yield: $82 \%$; m.p.: 200-202 ${ }^{\circ} \mathrm{C}$; IR (KBr, $\left.v_{\max }, \mathrm{cm}^{-1}\right): 3043(\mathrm{Ar}-\mathrm{H}), 2978(\mathrm{C}-\mathrm{H}$, $\left.\mathrm{CH}_{2}\right), 1745(\mathrm{C}=\mathrm{O}), 689(\mathrm{C}-\mathrm{S}), 1175(\mathrm{C}-\mathrm{N}), 1678(\mathrm{C}=\mathrm{C}), 3330$ (N-H), $1551(\mathrm{~N}-\mathrm{O}) ;{ }^{1} \mathrm{H}$ NMR (300 MHz, DMSO): $\delta 1.18(\mathrm{t}$, $3 \mathrm{H}), 2.78(\mathrm{q}, 2 \mathrm{H}), 2.65(\mathrm{t}, 2 \mathrm{H}), 2.96(\mathrm{t}, 2 \mathrm{H}), 5.0(\mathrm{~s}, 1 \mathrm{H}), 6.22$ $(\mathrm{s}, 1 \mathrm{H}), 7.32(\mathrm{t}, 1 \mathrm{H}), 7.32(\mathrm{t}, 1 \mathrm{H}), 7.50(\mathrm{~d}, 1 \mathrm{H}), 7.53(\mathrm{~s}, 1 \mathrm{H})$, 7.69 (t, 1H), 7.68 (t, 1H), $7.75(\mathrm{t}, 1 \mathrm{H}), 7.95(\mathrm{~d}, 1 \mathrm{H}), 7.99(\mathrm{~d}$, $1 \mathrm{H}), 9.20(\mathrm{~s}, 1 \mathrm{H})$; MS m/z $448.49\left(\mathrm{M}^{+}\right)$; Elemental analysis (\%) calculated for $\mathrm{C}_{23} \mathrm{H}_{20} \mathrm{~N}_{4} \mathrm{O}_{4} \mathrm{~S}$ : C-61.59, H-4.49, N-12.49, O-14.27, S-7.15. Found C-61.02, H-4.01, N-11.98, O-13.98, S-7.12.

$\mathrm{N}$-(4-Benzoyl-5-ethylthiophen-2-yl)-3-(5,6-dichloro$\mathbf{1 H}$-benzo[d]imidazol-2-yl)propanamide (7c): Yield: $72 \%$; m.p.: $215-217{ }^{\circ} \mathrm{C}$; IR (KBr, $\left.\mathrm{n}_{\max }, \mathrm{cm}^{-1}\right): 3048$ (Ar-H), 2928 $\left(\mathrm{C}-\mathrm{H}, \mathrm{CH}_{2}\right), 1735(\mathrm{C}=\mathrm{O}), 679(\mathrm{C}-\mathrm{S}), 1155(\mathrm{C}-\mathrm{N}), 1678(\mathrm{C}=\mathrm{C})$, $3310(\mathrm{~N}-\mathrm{H}), 685(\mathrm{C}-\mathrm{Cl}) ;{ }^{1} \mathrm{H}$ NMR (300 MHz, DMSO): $\delta 1.28$ $(\mathrm{t}, 3 \mathrm{H}), 2.47(\mathrm{q}, 2 \mathrm{H}), 2.57(\mathrm{t}, 2 \mathrm{H}), 2.96(\mathrm{t}, 2 \mathrm{H}), 5.10(\mathrm{~s}, 1 \mathrm{H})$, $6.32(\mathrm{~s}, 1 \mathrm{H}), 6.51(\mathrm{~d}, 1 \mathrm{H}), 7.66(\mathrm{t}, 1 \mathrm{H}), 7.55(\mathrm{~s}, 1 \mathrm{H}), 7.89(\mathrm{t}$, $1 \mathrm{H}), 7.88$ (t, 1H), 7.99 (d, 1H), $8.15(\mathrm{~d}, 1 \mathrm{H}), 8.35$ (s, 1H), 8.45 (s, 1H), $9.20(\mathrm{~s}, 1 \mathrm{H})$; MS m/z $472.39\left(\mathrm{M}^{+}\right)$; Elemental analysis (\%) calculated for $\mathrm{C}_{23} \mathrm{H}_{19} \mathrm{~N}_{3} \mathrm{O}_{2} \mathrm{SCl}_{2}$ : C-58.48, H-4.05, N-8.90, O-6.77, S-6.79, Cl-15.01. Found C-57.98, H-3.98, N-8.01, O6.12, S-6.01, Cl-14.02.

\section{RESULTS AND DISCUSSION}

2-Amino-ethylthiophene (1) was treated at room temperature with phthalic anhydride after grinding both the reactants to a fine powder in a mortar for $1 \mathrm{~h}$ at ambient temperature (reaction monitored with TLC). It was extracted with aqueous bicarbonate solution. The clear filtrate on neutralization gave a product which was identified as 2-[(4-benzoyl-5-ethylthiphen2-yl)carbamoyl]benzoic acid (2) indicating that the anhydride ring had been opened in the solid state (Scheme-Ia). Similarly, 2-aminothiophene (1) was also treated with maleic anhydride and succinic anhydride under similar conditions, which led to the formation of 2-[(4-benzoyl-5-ethylthiophen-2-yl)carbamoyl]-acrylic acid (4) and propionic acid (6), respectively (Scheme-Ib and Ic). The products 2, 4 and 6 were characterized by elemental analysis (Table-1) and by IR, ${ }^{1} \mathrm{H}$ NMR and MS spectroscopy (Table-2). The foregoing 2-[(4benzoyl-5-ethyl-thiphen-2-yl)carbamoyl]benzoic acid (2) was subjected to cyclocondensation with $o$-phenylenediamine in pyridine to give 2-(1H-benzo[d]imidazolyl-2-yl)- $N$-(4benzolyl-5-ethylthiophen-2-yl))benzamides (3) (Scheme-Ia). Similarly, 2-[(4-benzoyl-5-ethylthiophen-2-yl)carbamoyl]acrylic acid (4) and propionic acid (6) were cyclized to the corresponding benzimidazoles $\mathbf{5}$ and $\mathbf{7}$, respectively by refluxing them in pyridine solvent separately (Scheme-Ib and Ic). The structures of $\mathbf{3 , 5}$ and $\mathbf{7}$ were identified by analytical (Table-1) and spec-troscopic (IR, ${ }^{1} \mathrm{H}$ NMR and MS) data (Table-2).

The newly synthesized intermediates, viz. 2-[(4-benzoyl5-ethylthiophen-2-yl)carbamoyl]benzoic acid (2), acrylic acid (4) and propionic acid (6) in their IR spectra exhibited two strong peaks around 3000 and $3200 \mathrm{~cm}^{-1}$ due to carboxyl $(\mathrm{OH})$ and amide $(\mathrm{NH})$ groups and two other strong absorptions at 1675 and $1695 \mathrm{~cm}^{-1}$ due to amide carbonyl and carboxyl carbonyl groups respectively. ${ }^{1} \mathrm{H}$ NMR spectra of the products

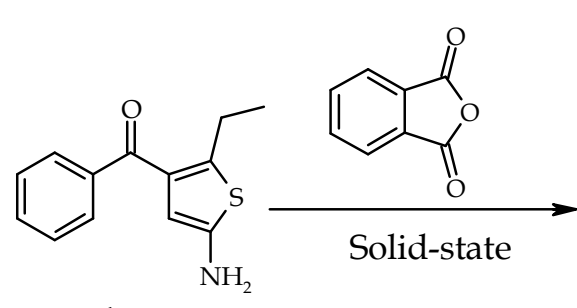

1

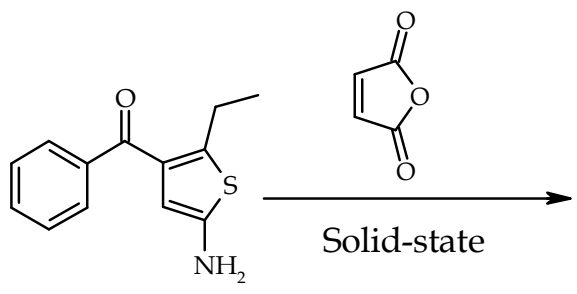

1<smiles>CCc1sc(NC(=O)c2ccccc2C(=O)O)cc1C(=O)c1ccccc1</smiles>

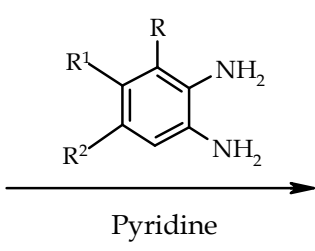

2

Scheme-Ia

$3 a=R, R^{1}, R^{2}=H$
$3 b=R=N_{2}, R^{1} \& R^{2}=H$
$3 c=R=H, R^{1} \& R^{2}=C l$

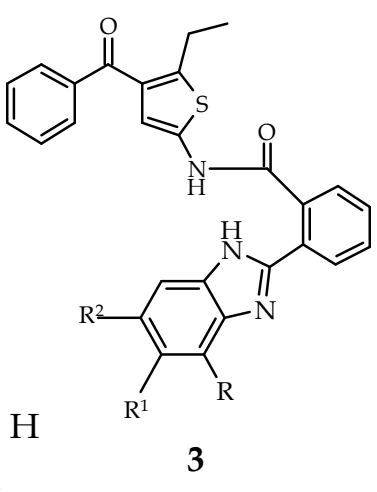

3
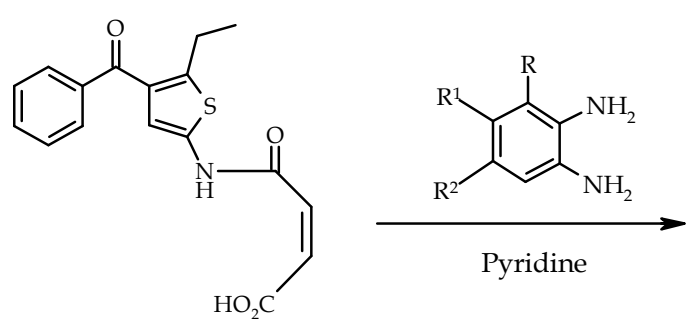

4

$$
\begin{aligned}
& 5 \mathbf{a}=\mathrm{R}, \mathrm{R}^{1}, \mathrm{R}^{2}=\mathrm{H} \\
& \mathbf{5 b}=\mathrm{R}=\mathrm{NO}_{2}, \mathrm{R}^{1} \& \mathrm{R}^{2}=\mathrm{H} \\
& 5 \mathrm{c}=\mathrm{R}=\mathrm{H}, \mathrm{R}^{1} \& \mathrm{R}^{2}=\mathrm{Cl}
\end{aligned}
$$

Scheme-Ib 


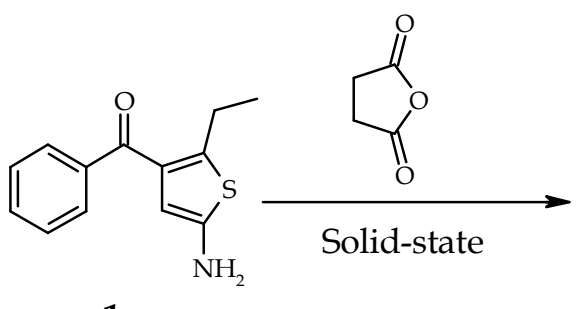

1<smiles>CCc1sc(NC(=O)CCC(=O)O)cc1C(=O)c1ccccc1</smiles>

6

Scheme-Ic

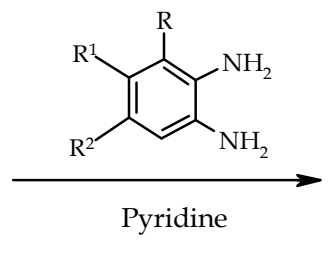

$7 \mathbf{a}=\mathrm{R}, \mathrm{R}^{1}, \mathrm{R}^{2}=\mathrm{H}$

$7 \mathrm{~b}=\mathrm{R}=\mathrm{NO}_{2}, \mathrm{R}^{1} \& \mathrm{R}^{2}=\mathrm{H}$

$7 \mathrm{c}=\mathrm{R}=\mathrm{H}, \mathrm{R}^{1} \& \mathrm{R}^{2}=\mathrm{Cl}$

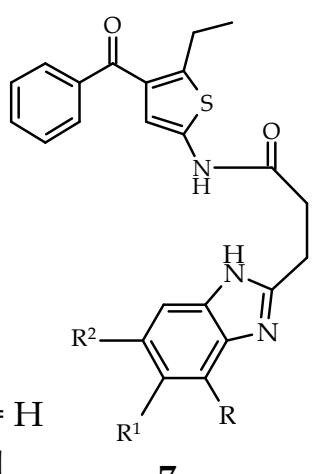

7
2, 4 and $\mathbf{6}$ showed down field signals at $\delta 9.7$ and 11.0 due to TABLE-1

ANTIBACTERIAL SCREENING RESULTS OF COMPOUNDS 3, 5 AND 7

\begin{tabular}{cccccc}
\hline \multirow{2}{*}{ Compd. } & Conc. & \multicolumn{4}{c}{ Zone of inhibition $(\mathrm{mm})$} \\
\cline { 3 - 6 } & $(\mu \mathrm{g} / \mathrm{mL})$ & $\begin{array}{c}E . \\
\text { coli }\end{array}$ & $\begin{array}{c}\text { P. } \\
\text { vulgaris }\end{array}$ & $\begin{array}{c}\text { B. } \\
\text { mycoides }\end{array}$ & $\begin{array}{c}\text { S. } \\
\text { aureus }\end{array}$ \\
\hline \multirow{2}{*}{ 3a } & 600 & 5.0 & 6.0 & 4.0 & 6.5 \\
& 900 & 9.0 & 10.0 & 12.0 & 13.5 \\
\hline \multirow{2}{*}{ 3b } & 600 & 4.5 & 5.0 & 3.5 & 6.0 \\
& 900 & 8.0 & 10.0 & 8.5 & 9.5 \\
\hline \multirow{2}{*}{ 3c } & 600 & 7.5 & 7.0 & 8.0 & 9.0 \\
& 900 & 9.5 & 11.0 & 10.0 & 12.0 \\
\hline \multirow{2}{*}{ 5a } & 600 & 6.0 & 8.0 & 5.0 & 6.0 \\
& 900 & 12.8 & 14.0 & 11.5 & 10.0 \\
\hline \multirow{2}{*}{ 5b } & 600 & 6.0 & 8.0 & 5.8 & 7.5 \\
& 900 & 10.5 & 13.5 & 12.0 & 11.0 \\
\hline \multirow{2}{*}{ 5c } & 600 & 5.5 & 6.0 & 7.5 & 9.0 \\
& 900 & 8.0 & 9.0 & 8.5 & 10.0 \\
\hline \multirow{2}{*}{ 7a } & 600 & 7.0 & 8.6 & 5.8 & 5.6 \\
& 900 & 13.0 & 15.0 & 12.5 & 14.0 \\
\hline \multirow{2}{*}{ 7b } & 600 & 5.5 & 6.0 & 4.5 & 6.5 \\
& 900 & 10.5 & 13.0 & 9.0 & 10.0 \\
\hline \multirow{2}{*}{ 7c } & 600 & 7.0 & 8.0 & 5.5 & 7.0 \\
& 900 & 9.5 & 11.0 & 11.0 & 10.5 \\
\hline \multirow{2}{*}{ Streptomycin } & 9.0 & 7.5 & 5.0 & 3.0 \\
& & 11.5 & 10.0 & 7.0 & 6.0 \\
\hline \multirow{2}{*}{} & & & & &
\end{tabular}

amide and carboxyl hydrogens, which are $\mathrm{D}_{2} \mathrm{O}$ exchangeable. The mass spectra of the products $\mathbf{2 , 4}$ and $\mathbf{6}$ agrees well with the proposed structures. Structures of the title compounds, viz., 2/3(1H-benzo[d]imidazol-2-yl)- $N$-(4-benzolyl-5-ethylthiophene-2-yl)benzamides (3), acrylamides (5) and propionamides (7) were also established by IR, ${ }^{1} \mathrm{H}$ NMR and MS spectra data (Table-2). IR spectra of $\mathbf{3}, \mathbf{5}$ and $\mathbf{7}$ showed absorptions around $3400,3290,1685 \mathrm{~cm}^{-1}$ due to amide carbonyl, amide $\mathrm{NH}$ and benzimidazole $\mathrm{NH}$ respectively. ${ }^{1} \mathrm{H}$ NMR spectra of $\mathbf{3}, \mathbf{5}$ and $\mathbf{7}$ conspicuously shown two down field signals at $\delta$ 9.7 and 11.0 due to the amide and newly formed benzimidazole ring $\mathrm{NH}$ protons, respectively. The product structures were further confirmed by their mass spectra.

Antibacterial activity: 2/3(1H-benzo[ $d]$ imidazol-2-yl)$N$-(4-benzolyl-5-ethylthiophene-2-yl)benzamides (3), acrylamides (5) and propionamides (7) were screened for their antibacterial activity against two Gram-negative bacteria Escherichia coli and Proteus vulgaris and two Gram-positive bacteria Bacillus mycoides and Staphylococcus aureus at 600 and $900 \mu \mathrm{g} / \mathrm{mL}$ concentration using the filter paper disc tech- nique of Vincent et al. [12]. The zone of inhibition formed was TABLE-2

\section{ANTIFUNGAL SCREENING RESULTS OF} COMPOUNDS 3, 5 AND 7

\begin{tabular}{|c|c|c|c|}
\hline \multirow{2}{*}{ Compd. } & \multirow{2}{*}{$\begin{array}{l}\text { Conc. } \\
(\mu \mathrm{g} / \mathrm{mL})\end{array}$} & \multicolumn{2}{|c|}{ Spore germination inhibition $(\%)$} \\
\hline & & D. halides & F. oxysporum \\
\hline \multirow{4}{*}{$3 \mathbf{a}$} & 160 & 270 & 27.5 \\
\hline & 320 & 59.6 & 96.0 \\
\hline & 480 & 91.0 & 100.0 \\
\hline & 640 & 100.0 & 100.0 \\
\hline \multirow{4}{*}{$\mathbf{3 b}$} & 160 & 17.0 & 30.0 \\
\hline & 320 & 70.0 & 97.0 \\
\hline & 480 & 94.5 & 100.0 \\
\hline & 640 & 100.0 & 100.0 \\
\hline \multirow{4}{*}{$3 c$} & 160 & 15.0 & 24.0 \\
\hline & 320 & 52.5 & 46.0 \\
\hline & 480 & 82.5 & 82.0 \\
\hline & 640 & 100.0 & 100.0 \\
\hline \multirow{4}{*}{$5 \mathbf{a}$} & 160 & 53.2 & 62.2 \\
\hline & 320 & 80.3 & 90.5 \\
\hline & 480 & 94.3 & 100.0 \\
\hline & 640 & 100.0 & 100.0 \\
\hline \multirow{4}{*}{$5 b$} & 160 & 28.6 & 21.1 \\
\hline & 320 & 54.0 & 47.6 \\
\hline & 480 & 68.9 & 72.0 \\
\hline & 640 & 100.0 & 100.0 \\
\hline \multirow{4}{*}{$5 c$} & 160 & 20.0 & 19.2 \\
\hline & 320 & 39.5 & 28.0 \\
\hline & 480 & 76.2 & 66.0 \\
\hline & 640 & 100.0 & 85.1 \\
\hline \multirow{4}{*}{$7 \mathbf{a}$} & 160 & 27.3 & 62.0 \\
\hline & 320 & 53.2 & 95.0 \\
\hline & 480 & 90.3 & 100.0 \\
\hline & 640 & 100.0 & 100.0 \\
\hline \multirow{4}{*}{$7 \mathbf{b}$} & 160 & 80.5 & 55.5 \\
\hline & 320 & 100.0 & 79.0 \\
\hline & 480 & 100.0 & 95.8 \\
\hline & 640 & 100.0 & 100.0 \\
\hline \multirow{4}{*}{$7 c$} & 160 & 17.3 & 30.5 \\
\hline & 320 & 36.6 & 49.8 \\
\hline & 480 & 58.2 & 79.0 \\
\hline & 640 & 100.0 & 86.0 \\
\hline
\end{tabular}

measured in mm. Streptomycin antibiotic disc (High media SD 181) was used as standard for comparison.

Table-1 reveals that the compounds of the series $\mathbf{3 , 5}$ and 7 exhibited appreciable activity against both Gram-positive and Gram-negative bacteria. In this series, the compounds $\mathbf{5 a}$ and 7a possessing acrylamide and propionamide groups with unsubstituted benzimidazole moieties showed maximum 
activity by inhibiting growth of all the bacteria to a significant level in comparison with the standard drug streptomycin at the same concentration $(900 \mu \mathrm{g} / \mathrm{mL})$. Hence, they can be exploited for formulation of bacterocides.

Antifungal activity: 2/3(1H-benzo[ $d]$ imidazol-2-yl)- $N$ (4-benzolyl-5-ethylthiophene-2-yl)-benzamides (3), acrylamides (5) and propionamides (7) were also screened for their antifungal activity against two plant pathogens viz., Fusarium oxysporum and Drescherla halides following the glass slide humid chamber technique [13] at $160 \mu \mathrm{g}, 320 \mu \mathrm{g}, 480 \mu \mathrm{g}$ and $640 \mu \mathrm{g} / \mathrm{mL}$ concentration. Mancozeb (fungicide) was used as the standard for comparison of the activity.

From Table-2, it is evident that these compounds exhibit strong fungicidal activity, which was lethal even at $480 \mu \mathrm{g} / \mathrm{mL}$ concentration. In this series, the compounds $\mathbf{3 a}, \mathbf{5 a}, \mathbf{7 a}$ and $\mathbf{7 b}$ exhibited remarkable fungitoxicity against both the fungi. This may be due to the presence of the benzimidazole ring-carrying isoxazolyl benzamide, acrylamide and propionamide moieties and also may be due to presence of the nitro group (in compound 7b). Hence, they can be exploited for formulation of fungicides (Table-2).

\section{Conclusion}

Syntheses of novel isoxazolyl benzoimidazolyl benzamides, acrylamides and propionamides have been achieved in a two-step process, in which the first step is a solid state reaction. The antimicrobial screening results indicated that the compounds $5 \mathbf{a}$ and $7 \mathbf{a}$ are found to be active agents and can be exploited for formulation of bacterocides and fungicides.

\section{REFERENCES}

1. P.N. Preston, Chem. Rev., 74, 279 (1974); https://doi.org/10.1021/cr60289a001.

2. R. Cedillo-Rivera and O. Munoz, J. Med. Microbiol., 37, 221 (1992); https://doi.org/10.1099/00222615-37-3-221.

3. B. Chavez, R. Cedillo-Rivera and A. Martinez-Palomo, J. Protozool., 39, 510 (1992); https://doi.org/10.1111/j.1550-7408.1992.tb04841.x.

4. G. Navarrete-Vázquez, R. Cedillo, A. Hernández-Campos, L. Yépez, F. Hernández-Luis, J. Valdez, R. Morales, R. Cortés, M. Hernández and R. Castillo, Bioorg. Med. Chem. Lett., 11, 187 (2001); https://doi.org/10.1016/S0960-894X(00)00619-3.

5. H. Yun, J. Yang, B. Wu, L. Risen and E.E. Swayze, Bioorg. Med. Chem. Lett., 14, 1217 (2004); https://doi.org/10.1016/j.bmcl.2003.12.051.

6. M.A. Weidner-Wells, K.A. Ohemeng, V.N. Nguyen, S. Fraga-Spano, M.J. Macielag, H.M. Werblood, B.D. Foleno, G.C. Webb, J.F. Barrett and D.J. Hlasta, Bioorg. Med. Chem. Lett., 11, 1545 (2001); https://doi.org/10.1016/S0960-894X(01)00024-5.

7. P.P. Seth, E.A. Jefferson, L.M. Risen and S.A. Osgood, Bioorg. Med. Chem. Lett., 13, 1669 (2003); https://doi.org/10.1016/S0960-894X(03)00245-2.

8. M. Nakanishi, T. Tahara, K. Araki, M. Shiroki, T. Tsumagari and Y. Takigawa, J. Med. Chem., 16, 214 (1973);

https://doi.org/10.1021/jm00261a010.

9. S. Tinney, J.P. Sanchez and J.A. Nogas, J. Med. Chem., 17, 624 (1974); https://doi.org/10.1021/jm00252a011.

10. H. Nagaoka, H. Hara and T. Mase, Heterocycles, 31, 1241 (1990); https://doi.org/10.3987/COM-90-5442.

11. P.A.M. van der Klein, A.P. Kourounakis and A.P. IJzerman, J. Med. Chem., 42, 3629 (1999); https://doi.org/10.1021/jm991051d.

12. J.C. Vincent, H.N. Vincent and J. Morton, Proc. Soc. Exp. Biol. Med., 55, 162 (1944); https://doi.org/10.3181/00379727-55-14502.

13. E.H. Allen and J. Kuc, Phytopathology, 58, 776 (1968). 